\title{
Optically Driven Domain Instability and High-Frequency Current Oscillations in Photoexcited GaAs under Nonuniform Electron Heating
}

\author{
L. SubaČIUS* AND I. KašAlynaS \\ Semiconductor Physics Institute, A. Goštauto 11, 01108 Vilnius, Lithuania \\ Fast domain instabilities induced by light-interference pattern in \\ dc-biased semi-insulating GaAs are investigated. Current oscillations in \\ GHz-frequency range are observed due to nonuniform electron heating and \\ domains formation in light-induced grating. Characteristic features of the \\ oscillations under various experimental conditions are presented. Numerical \\ calculations based on the hot-electron hydrodynamic model are used to ex- \\ plain the observed nonlinear features under various external bias and periods \\ of the grating.
}

PACS numbers: 71.55.Eq, 72.20.Ht

\section{Introduction}

Light-triggered domain instability and optically nonlinear features under nonuniform carrier heating in photorefractive semiconductors with negative differential resistivity (NDR) is an important current research problem, implicating potential applications in high-speed microelectronics and optoelectronics. Application of strong electric field and spatially nonuniform illumination results in novel nonlinear phenomena in semiconductor, e.g., the domain instabilities and photocurrent oscillations at $\mathrm{GHz}$ frequencies $[1,2]$. Recent theoretical investigations revealed a possibility for enhancement of the response of nonlinear system and generation of the oscillating current pulses from the NDR semiconductor under excitation by light-interference pattern [3-5]. Nonlinear response of the system can be very complex, and one can obtain several types of the domain instabilities:

*corresponding author; e-mail: liudas@pfi.lt 
periodic with the fundamental frequency, periodic with a number of harmonics, and chaotic [3].

We present the results of experimental and numerical study of light-interference pattern governed hot-electron nonlinearity in dc-biased semi-insulating GaAs crystal. We show that nonuniform carrier heating in transient grating leads to domain instabilities and photocurrent oscillations in $\mathrm{GHz}$ frequency range. The oscillations arising in strong external dc field (above $3 \mathrm{kV} / \mathrm{cm}$ ) are related with the NDR region in GaAs crystal. Their frequency depends on the spatial period of interference pattern created by laser beams. In this paper the most important properties of instability of this kind are presented along with numerical calculations based on the hydrodynamic approach.

\section{Results and discussion}

We analyse the case where spatially modulated free carrier (FC) plasma is photoexcited from the deep donor levels and band-to-band transitions in dc-biased GaAs crystal. A schematic diagram of experimental setup is shown in Fig. 1a. The Nd:YAG laser pulses of duration $\tau \approx 4$ ns and wavelength of

(a)
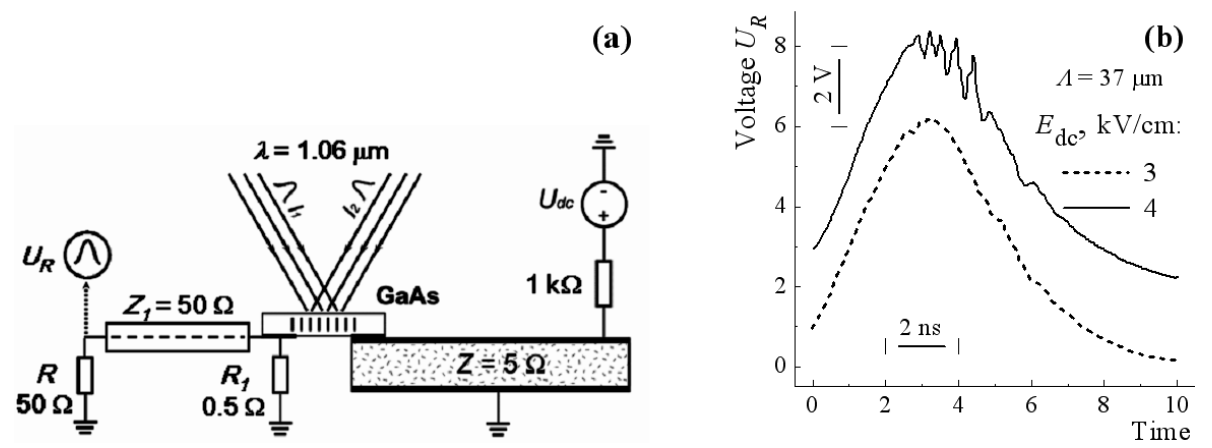

Fig. 1. (a) Experimental setup for observation of GHz-frequency photocurrent oscillations, induced by interference pattern in dc-biased GaAs. (b) Response $U_{R}$, measured on load resistor $R$ at grating spacing $\Lambda=37 \mu \mathrm{m}$ and external dc-field $E_{\mathrm{dc}}, \mathrm{kV} / \mathrm{cm}$ : 3 (dash line) and 4 (solid line).

$\lambda=1064 \mathrm{~nm}$ with beams intensity $I_{1} \cong I_{2}$ interfere inside the GaAs crystal, creating a light-interference pattern of intensity $I_{0}=\left(I_{1}+I_{2}\right)[1+m \cos (2 \pi x / \Lambda)]$ with spatial period $\lambda$ and modulation depth $m \cong 1$. The incident interference pattern causes generation of FC grating and as a result electric field grating in the sample. Transient gratings with the spacing $\Lambda=20-50 \mu \mathrm{m}$ were recorded in a bulk of dc-biased GaAs. Results are presented at photoexcited FC concentration of about $10^{16} \mathrm{~cm}^{-3}$ and applied dc-voltage $U_{\mathrm{dc}}$ up to $1 \mathrm{kV}$. 
The 75-155 $\mu \mathrm{m}$ thick samples with annealed planar Au/Ge/Ni contacts were prepared from semi-insulating GaAs wafer (dark resistivity about $10^{7} \Omega \mathrm{cm}$ ). They were mounted in series with a long segment (of about $2 \mathrm{~m}$ ) of a low-impedance $(Z \approx 5 \Omega)$ strip line, initially charged to the voltage $U_{\mathrm{dc}}$, providing an average external dc-field in the illuminated crystal, $E_{\text {ext }}(t)$, equal to

$$
E_{\mathrm{ext}}(t)=E_{\mathrm{dc}} \frac{R_{\mathrm{s}}(t)}{R_{\mathrm{s}}(t)+Z+R_{1}} .
$$

Here $E_{\mathrm{dc}}=U_{\mathrm{dc}} / l$ is the dc-field before illumination, $l=0.5-1 \mathrm{~mm}$ is the distance between planar electrodes of the sample, $R_{\mathrm{s}}(t)$ and $R_{1}=0.5 \Omega$ are the resistivity of the sample and loading resistor, respectively. The voltage $U_{R}(t)$ across the resistor $R=50 \Omega$ was measured by real-time oscilloscope (see Fig. 1a). The bandwidth frequency of this photoconductivity measurement system was of about 3-5 GHz. In this way, the experimental technique allowed us to monitor GHz-frequency photocurrent oscillations (see Fig. 1b) stimulated by the laser beams in dc-biased GaAs due to nonuniform carrier heating in the NDR region.
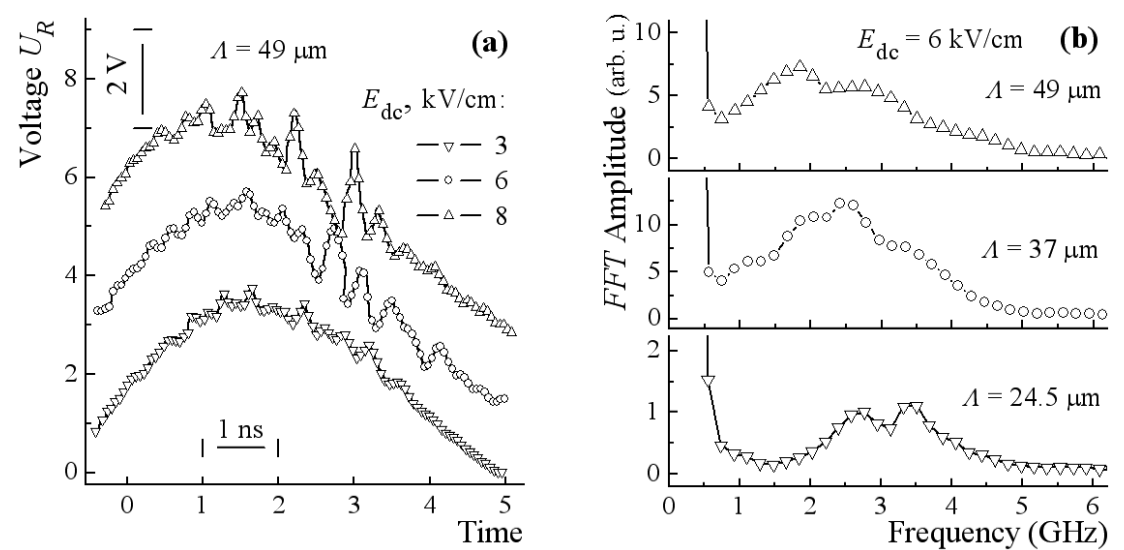

Fig. 2. (a) Dynamics of the response $U_{R}$, measured in dc-biased GaAs at excitation by interference pattern with spacing $\Lambda=49 \mu \mathrm{m}$ and at various applied fields. (b) Fourier spectrum of $U_{R}(t)$ at applied field $E_{\mathrm{dc}}=6 \mathrm{kV} / \mathrm{cm}$ and at different values of the grating spacing.

Monitoring of the domain instabilities in dc-biased transient grating is carried out by varying the external electric field and spacing of interference pattern. Figure 2a demonstrates typical photocurrent traces of the $49 \mu \mathrm{m}$ period grating, obtained at different external dc-fields. We note that the oscillating behavior occurs at the end of photoexcitation and arises only in the NDR region (i.e., at $E_{\mathrm{dc}} \geq 3 \mathrm{kV} / \mathrm{cm}$, see Figs. $1 \mathrm{~b}$ and $2 \mathrm{a}$ ). Next important result is achieved from the Fourier spectrum analysis, varying the spacing of interference pattern. This is demonstrated in Fig. 2b. The obtained results point out the oscillation frequency 
dependence on the spacing of interference pattern. It is seen that the oscillation frequency decreases when the grating spacing increases. This important feature can be understood as a consequence of high-field domains propagation across the optically modulated FC structure: the oscillation frequency corresponds to a time required for the domain to drift a distance which equals the grating spacing. In this way, the experimental evidence of the phenomena of oscillating current in light-induced grating confirms previous results of the picosecond four-wave mixing in dc-biased GaAs [6]. Next, the observed phenomena are simulated numerically for conditions close to those experimentally realized.

For the simulation a hydrodynamic model based on the conservation equations of hot-carrier velocity and mean energy is applied by using the conventional parameters of semi-insulating GaAs:EL2 crystal at $\lambda=1.06 \mu \mathrm{m}[5,6]$. In Fig. 3
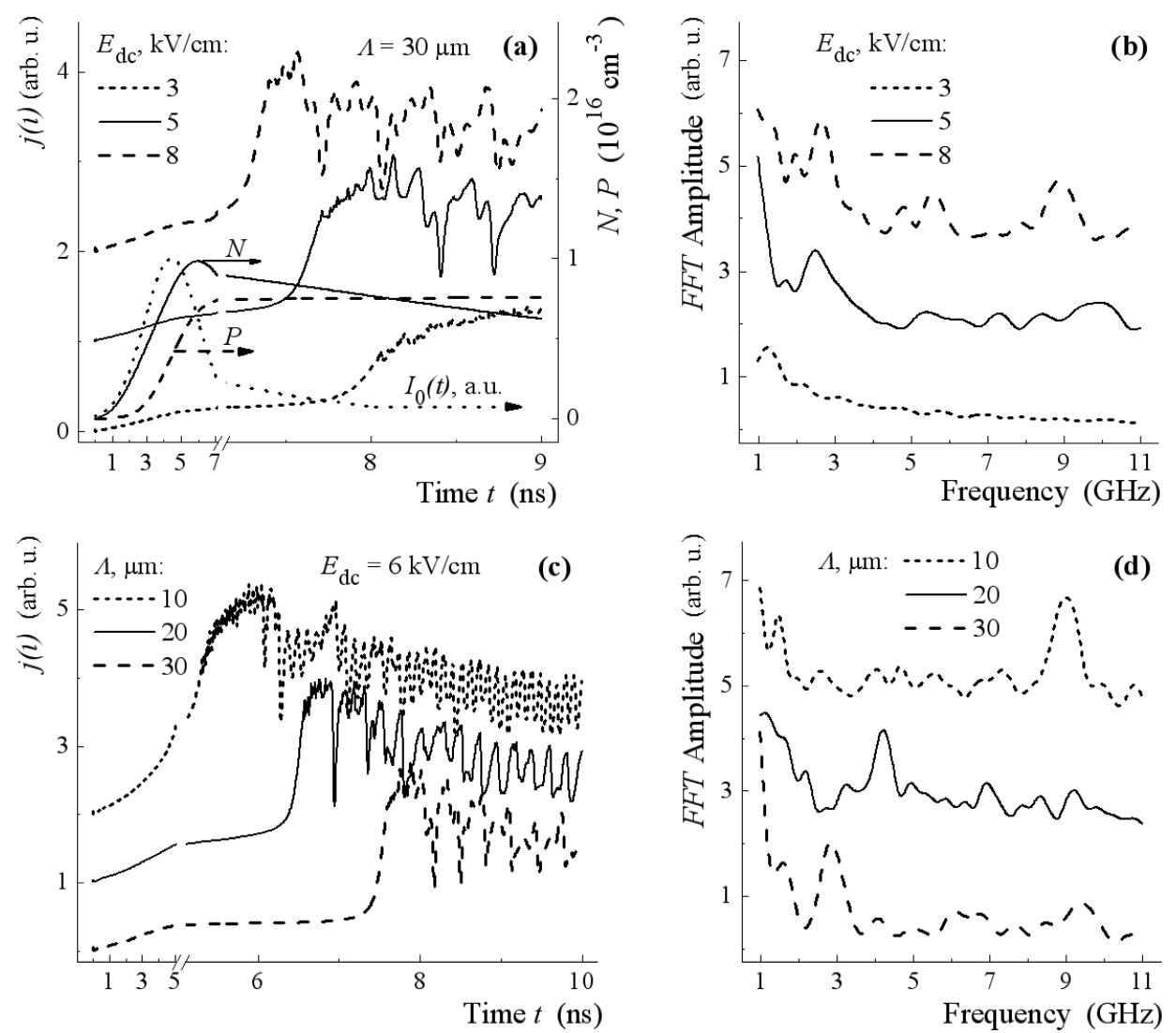

Fig. 3. Calculated dynamics of the current density $j(t)$ (left-hand side column) and its Fourier spectrum for time interval from 5 ns to 10 ns (right-hand side column) in dc-biased GaAs: (a),(b) at interference pattern with spacing $\Lambda=30 \mu \mathrm{m}$ and at various applied fields; (c),(d) at applied field $E_{\mathrm{dc}}=6 \mathrm{kV} / \mathrm{cm}$ and at different values of the grating spacing. Right-hand scale in (a) shows time dependence of the excitation intensity $I_{0}(t)$ and density of electrons $N$ and holes $P$ used in calculation. 
we plotted the dynamics of photocurrent density $j(t)$ and corresponding Fourier spectrum (FFT) of current density, calculated for various external bias and grating spacing at ns-laser excitation. In addition, the calculated dynamics of generated carrier densities $(N$ and $P)$ is presented in Fig. 3a. It is seen that the calculation of the current dynamics in dc-biased nonuniformly photoexcited GaAs confirms the formation of oscillating current effect. The effect appears after the photoexcitation at $E_{\mathrm{dc}} \geq 3 \mathrm{kV} / \mathrm{cm}$ and depends on the grating spacing, as it was shown experimentally.

More detailed analysis of carrier-field spatio-temporal distributions indicated that dynamics of nonuniform electron heating and the domains formation is quite complicated and depicts various nonlinear phenomena. We note that the origin of the oscillating current is associated with the formation of traveling domain structure in dc-biased GaAs under nonuniform illumination. The period of oscillations corresponds to domain drift between the grating peaks, i.e., the oscillation frequency decreases, if grating spacing increases.

To summarize, we show experimentally and numerically that nonlinear transport and ultrafast hot-electron dynamics in optically modulated GaAs crystal leads to domain instabilities and oscillating behavior of the current density in GHz-frequency range. Phenomenon of the oscillating current is attributed to formation of light-triggered high-field domain structure, which propagates in light-induced FC grating. This investigation revealed novel possibility for the external control of the oscillation frequency by varying the spacing of incident interference pattern.

\section{References}

[1] M. Segev, B. Collings, D. Abraham, Phys. Rev. Lett. 76, 3798 (1996).

[2] L. Subačius, K. Jarašiūnas, E. Starikov, V. Gružinskis, P. Shiktorov, Phys. Status Solidi B 204, 473 (1997).

[3] L.L. Bonilla, M. Kindelan, P.J. Hernando, Phys. Rev. B 58, 12844 (1998).

[4] Y.-H. Shiau, Phys. Rev. B 60, 15534 (1999).

[5] L. Subačius, E. Starikov, P. Shiktorov, V. Gružinskis, K. Jarašiūnas, J. Opt. Soc. Am. B 15, 2045 (1998).

[6] L. Subačius, I. Kašalynas, R. Aleksiejūnas, K. Jarašiūnas, Appl. Phys. Lett. 83, 1557 (2003). 